\title{
Effectiveness of Implementing Process Improvement Strategies on Turnaround Time of Emergency Investigations, in Clinical Biochemistry Laboratory
}

\author{
AS Shilpasree ${ }^{1}, K$ R Pravin Chandra ${ }^{2}$, Vidya S Patil ${ }^{3}$, Shrirang P Kulkarni ${ }^{4}$, \\ Rakesh T Muddaraddi ${ }^{5}$, Vijayetha P Patil ${ }^{6}$, Deepti G Ingleshwar ${ }^{7}$
}

\begin{abstract}
Background: Turnaround time (TAT) is one of the most noticeable signs of a laboratory service and is used by many clinicians as a benchmark to judge the quality of laboratory service. Despite automation and computerisation, delayed TAT of emergency department (ED) samples was a complaint received in our clinical biochemistry laboratory. After process mapping and root cause analysis, we designed an improvement strategy which included the introduction of emergency request forms (ERF), creating awareness in the technical staff by proper training and streamlining the work flow path.

Aim: To compare the TAT of ED samples for electrolytes, received 1 month before and after implementing the process improvement strategy. Materials and methods: Data regarding the number of electrolyte assays ordered from ED for 1 month before and after implementing the improvement strategy was obtained from hospital laboratory information system (LIS). TAT was calculated as the interval between sample accession in the laboratory and the time results were reported in LIS. Independent sample ' $t$ ' test was used to compare the mean TAT before and after the intervention
\end{abstract}

Results: Independent sample 't' test showed reduction in the TAT of ED electrolyte assays after intervention compared to before intervention (78.74 and 110.08 min respectively) with a statistically significant $(p<0.001)$ difference of $31.34 \mathrm{~min}$.

Conclusion: Significant reduction in the TAT of ED samples after intervention highlighted the power of analytical phase intervention in the form of reallocation of resources and staff awareness in effectively reducing the TAT. The study also proved the usefulness of having separate colour coded ERF in early identification, segregation and processing of ED samples.

Clinical Significance: Decreased laboratory turnaround time of ED investigations is clinically significant because along with customer satisfaction it also improves the efficiency of work by allowing the early diagnosis, treatment, patient discharge and provides a way of cost cutting.

Keywords: Automation, Emergency request forms, Laboratory information system, Turnaround time.

Indian Journal of Medical Biochemistry (2019): 10.5005/jp-journals-10054-0105

\section{INTRODUCTION}

Quality of laboratory service is assessed by the availability, reliability (accuracy and precision), cost and timeliness of the tests. Clinicians often expect a rapid, reliable and efficient service delivered at low cost. ${ }^{1}$ Laboratory tests make a major impact on clinical decision making especially when the clinicians are left with diagnostic uncertainty after the initial clinical assessment and guide them to adopt proper management strategies. ${ }^{2}$ As the test results are crucial in making the definitive diagnosis and management plan, patient outcome may rely upon prompt reporting of the tests. ${ }^{3}$ When the patients present to emergency department (ED) with severe illness and significant risk of rapid progression of disease, or when the decision to admit the patient and treat immediately requires further risk assessment, the ED clinician often depends upon the laboratory test results. ${ }^{4}$ In these situations, timeliness is perhaps the most important characteristic to the clinician compared to the analytical performance and cost. This preference has led to the widespread use of point of care testing (POCT) analysis nowadays.

Turnaround time (TAT) is one of the most noticeable signs of a laboratory service and is used by many clinicians as a benchmark to judge the quality of laboratory service. Literature reveals a variety of definitions of TAT based on the test ordered, priority

\author{
1,5,6 Associate Professor, ${ }^{2}$ Epidemiologist and Biostatistician, \\ ${ }^{3}$ Professor and Head, ${ }^{4}$ Professor, ${ }^{7}$ Assistant Professor \\ ${ }^{1,3-7}$ Department of Biochemistry, SDM College of Medical Sciences and \\ Hospital, Dharwad, Karnataka, India \\ ${ }^{2}$ Department of Community Medicine, SDM College of Medical \\ Sciences and Hospital, Dharwad, Karnataka, India
}

Corresponding Author: AS Shilpasree, Associate Professor, Department of Biochemistry, SDM Collage of Medical Sciences and Hospital Dharwad, Karnataka, India, e-mail: shilpasree2007@gmail.com How to cite this article: Shilpasree AS, Pravin Chandra KR, Patil VS, Kulkarni SP, Muddaraddi RT, Patil VP, Ingleshwar DG. Effectiveness of Implementing Process Improvement Strategies on Turnaround Time of Emergency Investigations, in Clinical Biochemistry Laboratory. Indian J Med Biochem 2019;23(2):287-292.

Source of support: Nil

Conflict of interest: None

and population served. According to CAP Q probe assay, there are differences between clinicians and laboratories in their definition of TAT. ${ }^{1}$ The term therapeutic TAT is used to describe the interval between the test order and the time when treatment decision is made. Perhaps many steps in the total testing cycle occur outside the laboratory and are not under direct control of the laboratory

(0) The Author(s). 2019 Open Access This article is distributed under the terms of the Creative Commons Attribution 4.0 International License (https://creativecommons. org/licenses/by-nc/4.0/), which permits unrestricted use, distribution, and non-commercial reproduction in any medium, provided you give appropriate credit to the original author(s) and the source, provide a link to the Creative Commons license, and indicate if changes were made. The Creative Commons Public Domain Dedication waiver (http://creativecommons.org/publicdomain/zero/1.0/) applies to the data made available in this article, unless otherwise stated. 
person and most of the times data of extra laboratory activities are not readily available so it is not possible to use the therapeutic TAT. In such situations it is more convenient to use laboratory TAT which can be taken from the sample accession time in the laboratory to the time results are reported. ${ }^{5}$

Despite advances in the analytical technology, computerisation and transport system, delayed TAT is the most common complaint received by the laboratories and consumes much time and effort of the laboratory personnel in complaint resolution and service improvement. Unsatisfactory TAT in the emergency department elicits immediate response and labelled as poor performance of the laboratory.

Delayed TAT of ED samples was one of the complaints received in our clinical biochemistry laboratory which was more in morning hours (between 6 am to $9 \mathrm{am}$ ). After the root cause analysis, this delay was attributed to the fact that most of the samples (both from general ward and ED) arrive to the lab in morning hours especially between 6am to 9am and availability of less technical staff during this time of the day. It was found difficult and time consuming for the laboratory staff to segregate the ED samples from routine samples. We aimed to improve the efficiency of test reporting and reduce the TAT by application of lean six sigma improvement tools and focused primarily on identification and elimination of non value added processes like sample handling by multiple technicians and multiple checks at different stations. With this rationale we designed a new strategy which included

a. Introducing the new colour coded emergency request forms which aids in the early identification, processing and reporting of emergency samples

b. Proper training of laboratory technical staff about the importance of TAT and methods which can be adopted in the laboratory to reduce the ED TAT and

c. Streamlining the laboratory workflow

We hypothesise that, by reallocation of resources and staff motivation it is possible to reduce the TAT of ED samples. The aim of present study was to assess the difference in the TAT of ED electrolyte samples received 1 month before and 1 month after implementing the abovementioned strategy.

\section{Materials and Methods}

A retrospective study was conducted to compare the TAT of electrolyte assays requested from ED in clinical biochemistry laboratory, SDM College of medical sciences and hospital. Ethical committee clearance was obtained from the institutional ethical committee. Data regarding the number of electrolyte assays ordered for 1 month before implementing the improvement strategy was obtained from hospital laboratory information system (LIS). Delays due to instrument breakdown and hemolysed samples requiring repeat sampling were excluded from the study. We calculated the TAT of all electrolyte assays as the interval between sample accession in the laboratory and the time results were reported in LIS.

After the initial assessment of complete workflow, we applied lean six sigma improvement strategies to identify the potential bottlenecks in the work flow stream and used these inputs to design improvement tools. We introduced the coloured emergency request forms (in contrast to white routine request forms) which helps in the early identification and segregation of emergency samples from routine samples and circulated the forms only to the emergency departments (casualty, MICU, NICU, PICU and SICU). Few tests (which were not considered as emergency tests) were purposefully omitted from the new request forms only after consulting the clinicians to reduce the burden on laboratory. The new request forms comprised only $40 \%$ of the total tests in routine forms and in case the remaining tests were required, the ED staffs were given an option to send the routine form along with the emergency request form. Along with this, awareness was created in the laboratory staff regarding the importance of TAT on clinical decision making, patient length of stay, overall burden on hospital and patient. Proper training of technical staff was conducted on new work flow pattern. The technicians were instructed to identify the emergency request form as soon as it reaches the laboratory and analyse the sample on priority basis. The analytical steps occurring within the laboratory were streamlined accordingly.

- One technician was given the responsibility of emergency samples in each shift and instructed to make sure the results are reported within the defined TAT.

- The technical staff were informed to maintain a separate emergency register with the details of samples coming with ERF including the TAT and cause for delayed TAT if any.

- Separate sample acceptance station was made to immediately accept the samples coming with ERF

- Separate path was created for ED samples inside the laboratory so that they will not enter the routine analytical queue.

- It was instructed to centrifuge the ED samples on a separate centrifuge to avoid mixing of emergency samples with routine samples.

- ED samples were analysed exclusively in one chemistry analyser (Siemens dimension Rxl max) to avoid the overcrowding of samples while the routine samples were analysed on the other instrument.

- Priority was given to the emergency samples while reporting the results and technical staff was instructed to call the biochemist anytime regarding the clarification of results.

Laboratory workflow before and after the intervention and expected effects of intervention is represented in (Figs. 1 and 2)

No of electrolyte samples received from ED for 1 month after implementing the abovementioned strategy was obtained from hospital LIS and the mean TAT was calculated. Unpaired't' test was used to compare the mean TAT of ED electrolyte samples before and after the designed intervention. $p$ value $<0.05$ was considered statistically significant.

\section{Results}

A total 3448 electrolyte samples were analysed for the period of 1 month (before intervention) in clinical biochemistry laboratory which included the samples from ED as well as general wards coming with routine request forms (RRF). After excluding the delays due to instrument breakdown and hemolysed samples, number of electrolyte assays remained was 3344. Information on TAT was obtained from hospital LIS and mean TAT was calculated. The intervention was planned and implemented in a stepwise manner over the duration of 1 month. Number of electrolyte samples obtained from ED with emergency request forms (ERF) for the period of 1 month (after intervention) was 1001. After applying the abovementioned exclusion criteria, 962 assays were remained and mean TAT of these assays was calculated. IBM-SPSS-STATISTICSVersion 20 (licensed to SDM College of Medical Sciences, Dharwad) has been used for the statistical analysis. Descriptive statistics of study population (before and after the intervention) is given in (Table 1) and mean TAT before and after the intervention is shown in (Fig. 3). 


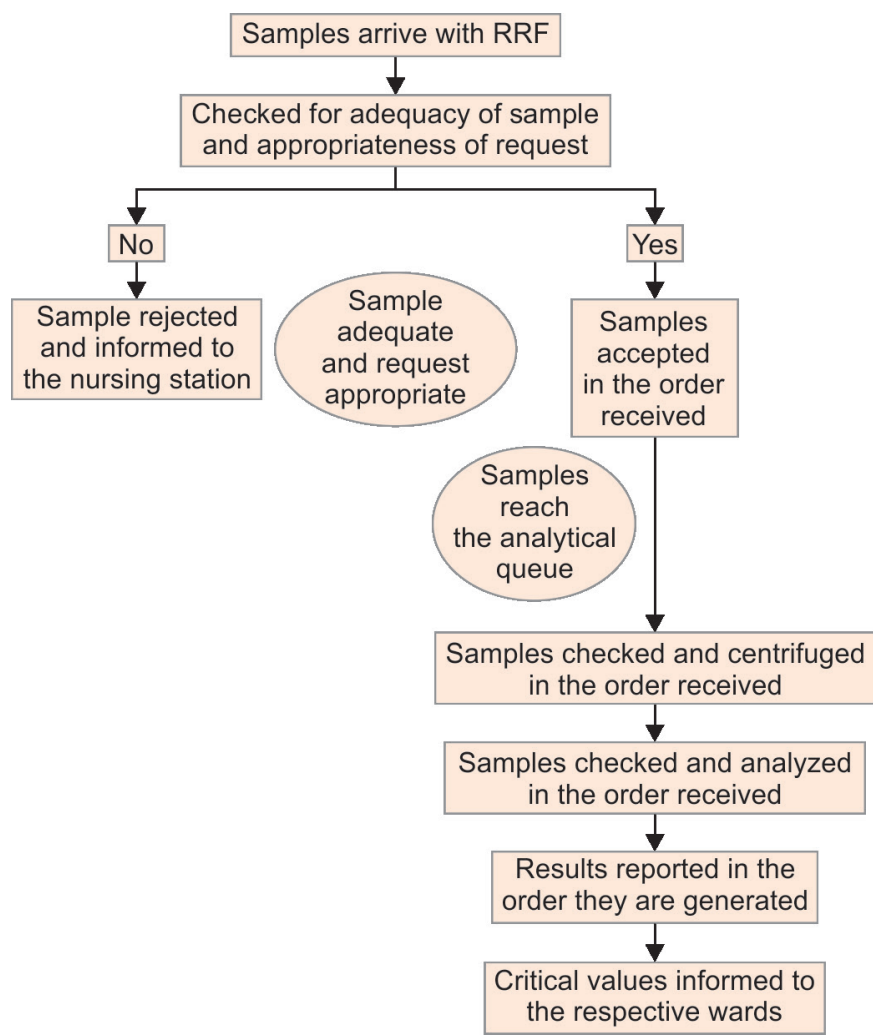

Fig. 1: Routine workflow of clinical biochemistry laboratory (before intervention)
The calculation of $5 \%$ trimmed mean demonstrates that, the mean approaches the median and hence it was meaningful to do trimming of extremes to remove the outliers. Trimming also gives the $95 \%$ confidence interval by taking the lower bound $2.5 \%$ and upper bound $97.5 \%$ quantiles.

Stem and leaf plots were observed to see the frequency with which the values appear in study population (Figs. 4 and 5). Box plot was constructed to show the variability among the values (Fig. 6). More variability was noticed outside the upper and lower quartile before intervention compared to after intervention. To overcome this variability, bootstrapping was done to estimate the statistical parameters (mean and 95\% confidence interval) by means of resampling with replacement. Since bootstrapping does not make any assumption about the distribution of sample, bootstrapped samples can be quite good approximates for population parameters. ${ }^{6}$ With a large sample size of 3344 (before intervention) and 962 (after intervention), in our study we have good estimates of population mean (110.08mins before intervention Vs $78.74 m i n s$ after intervention) and $95 \%$ confidence interval (107.66112.46mins before intervention Vs 76.31-81.24 mins after intervention).

Levene's test for equality of variances showed a significant difference in variances. Further the independent sample ' $t$ ' test yielded ' $t$ ' value of 17.90, degree of freedom (df) 2803.96 and significant difference between the mean TAT before and after intervention ( 31.336 mins, 95\% Cl: 27.90 to 34.76 mins) with a probability $p<0.001$. Hence it is concluded that, the difference of roughly 31 min between the TAT of ED electrolyte assays before and after intervention is statistically significant as well as practically important.

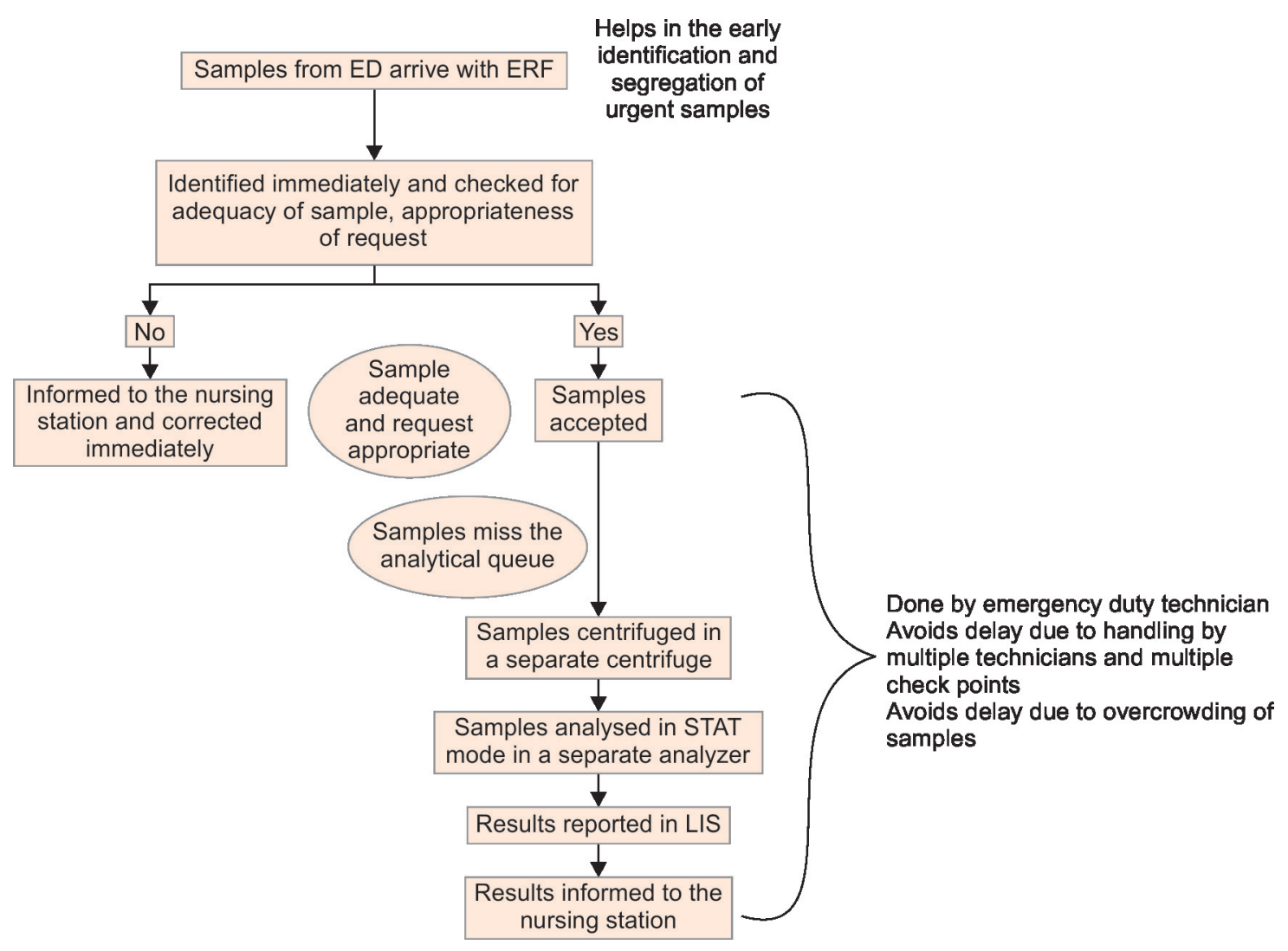

Fig. 2: Modified workflow of clinical biochemistry laboratory (after the intervention) and expected effects of intervention 
Table 1: Descriptive statistics of study population (before and after the intervention)

\begin{tabular}{l|l|l|}
\hline & Before & After \\
\hline Number $(\mathrm{n})$ & 3344 & 962 \\
Mean TAT in mins & 110.07 & $78.74^{*}$ \\
95\% confidence interval & $107.72-112.43$ & $76.24-81.23$ \\
Standard deviation & 69.55 & 39.45 \\
Median in mins & 102 & 73 \\
$5 \%$ trimmed mean in mins & 102.60 & 77.39 \\
\hline${ }^{*} p<0.001$ & &
\end{tabular}

\section{Discussion}

Implementing the best laboratory processes is crucial to attain the optimum quality of patient care. Although it looks simple, the lab operation is complex and time consuming due to the handling of large variety of samples both from inpatients and outpatients. Even after computerisation and automation of work flow, it is difficult to meet the customer satisfaction in terms of desired TAT. Since timeliness is the key performance indicator of laboratory efficiency and most important determining factor of patient care quality, it is important to monitor the laboratory TAT routinely.

Keeping in mind the increasing expectation of clinicians and continuously increasing workload on laboratory personnel, we designed a stepwise improvement strategy which included 5 stages: Define the problem, Analyze the existing workflow, Identify the lacunae, Improve the process by taking necessary interventions and Check the results. The intervention was designed using methods validated and accepted by different laboratories after doing necessary modification suitable to the present scenario. ${ }^{7,8}$

In the define phase, the problem was defined after discussion with treating clinicians. The most commonly encountered problem was delayed TAT of samples coming from ED especially in early

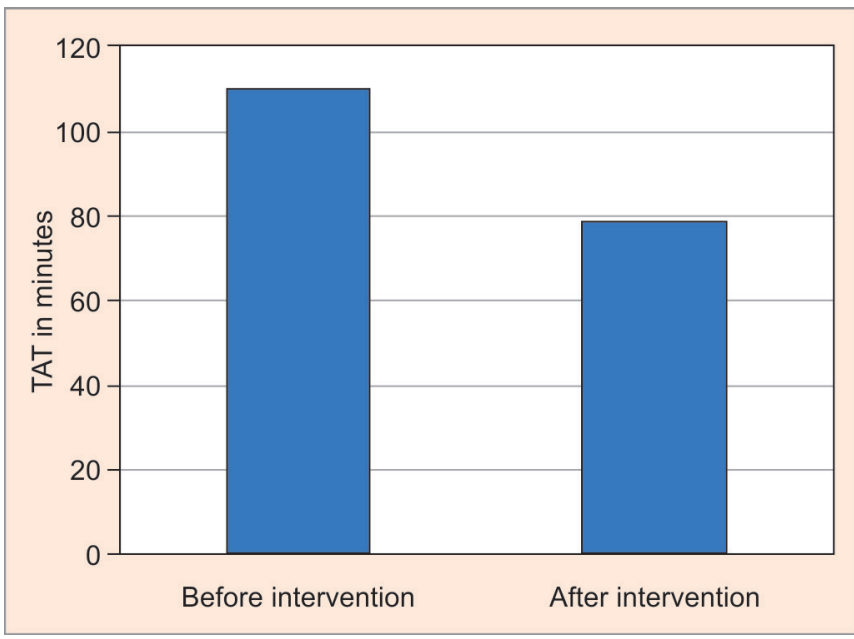

Fig. 3: Mean TAT before and after the intervention.

morning hours. In analyze phase, the entire process starting from clinicians ordering of test request till the availability of reports was mapped. The initial assessment revealed potential redundancies in both preanalytical and analytical phases. Since most of the processes in preanalytical phase take place outside the laboratory, the exact data is not available and these processes are not under the direct control of laboratory personnel, the shortcomings of pre analytical phase were ignored and analytical phase was concentrated. In the next phase, the lacunae in analytical phase were identified. The causes for delayed reporting of ED samples were identified as arrival of most of the samples both from ED and general wards in the morning hours (between 6 am to 9 am), difficulty to segregate ED samples from routine samples, lack

$\begin{array}{cl}\text { Frequency } & \text { Stem and Leaf } \\ 45.00 & 2.345667899 \& \\ 149.00 & 3.00111222333444555566667777788889999 \\ 205.00 & 4.000111111222223333333444445566666777788888889999 \\ 225.00 & 5.00000111111112222223333334444445556666777788888889999999 \\ 268.00 & 6.000000011111112222233333333444444555555556666666777777888889999999 \\ 285.00 & 7.000000001111112222222333333344444455555555566666667777778888889999999 \\ 192.00 & 8.0000011111222233334445555566667777888888999999 \\ 253.00 & 9.00000111111222222233333334444455555666666777778888888899999999 \\ 282.00 & 10.0000001111112222223333333444444555555666666777777778888888889999999 \\ 262.00 & 11.000000111111222222333333334444455555556666667777777888888999999 \\ 241.00 & 12.000000011111122222333333444444455555566666666777778888889999 \\ 214.00 & 13.0000000011111112222233334444445556666666777778889999 \\ 179.00 & 14.000000011112222333334444555566666778888999 \\ 121.00 & 15.0001112223334455566677889999 \\ 103.00 & 16.0001122333344455677788999 \\ 63.00 & 17.001223445667899 \\ 46.00 & 18.01233456679 \& \\ 34.00 & 19.0134578 \& \\ 28.00 & 20.234567 \& \\ 18.00 & 21.0579 \& \\ 15.00 & 22.14 \& \\ 9.00 & 23.01 \& \\ 107.00 \text { Extremes }(\geq 235)\end{array}$

Fig 4: Stem and leaf plot before intervention 

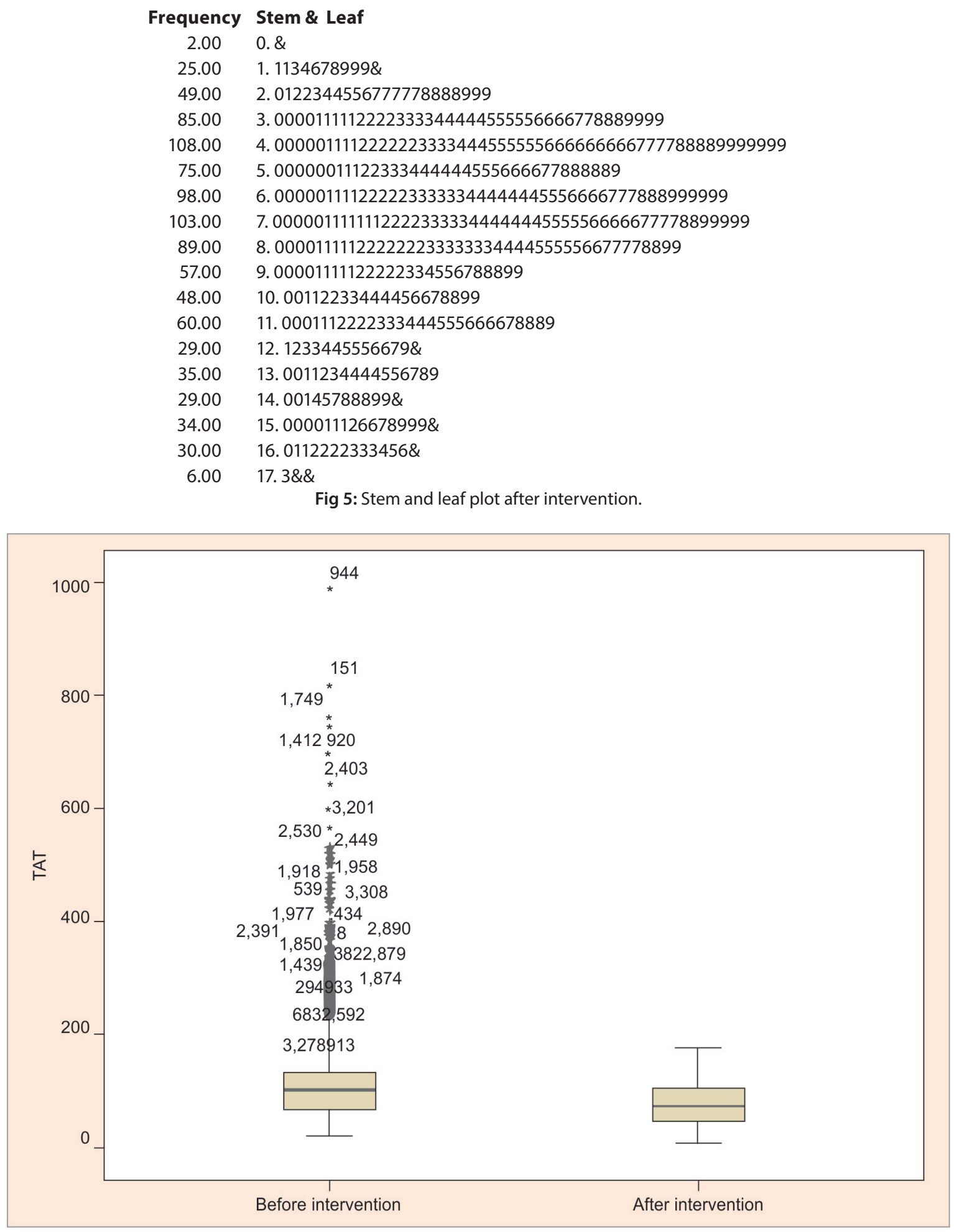

Before intervention After intervention

Fig 6: Box plot showing the TAT before and after intervention

of awareness in the technical staff regarding the importance of ED TAT and multiple checkpoints due to handling by multiple technicians. The lean six sigma principles were applied to design the improvement strategy after identifying the activities which consume unnecessary time and effort without any value addition (non value added processes). Non value added processes like sample handling from multiple technicians, unnecessary long work flow path and multiple check points at different stations were removed by redesigning the work flow path and keeping a separate technician to handle the emergency samples. New colour coded ERF were introduced, which helps in early identification and processing of emergency samples. Awareness was created among 
technicians regarding the importance of TAT on clinical decision making, patient length of stay, overall burden on hospital and patient. Proper training of technical staff was conducted on new work flow pattern. To check the results, this study was undertaken to compare the TAT of ED electrolyte assays before and after the intervention.

As a result of intervention, TAT of ED electrolyte assays was reduced from $110 \mathrm{~min}$ to $79 \mathrm{~min}$ ( total reduction of $31 \mathrm{~min}$ ) which is practically significant especially in ED as it allows the clinician to make quicker decision regarding patient admission or discharge, earlier and more appropriate diagnosis, fewer laboratory tests and shortened length of hospital stay. Although $70 \%$ of the total TAT is contributed by pre and post analytical phases and they need to be focused to reduce the overall TAT, changes can also be introduced during the analytical phase to reduce the sample process time. In our setup, this reduction in TAT after the analytical phase intervention highlighted the impact of workflow design, knowledge and awareness of technical staff on laboratory TAT. The study also proved the usefulness of having separate colour coded ERF in early identification, segregation and processing of ED samples. The findings were in accordance with study by Silvia Angeletti et al where gradual and step-by-step changes in sample management, staff adhesion and awareness led to significant reduction of TAT. ${ }^{9}$ According to Tzu-I Chien et al, prioritising the test performance and dedicated means of process control can significantly reduce the TAT of emergency tests without affecting the overall TAT. ${ }^{10}$ In a single centre intervention brought about by White BA et al, application of lean methodologies in the form of reallocation of resources without the addition of new resources and simple reorganisation of laboratory process flow effectively decreased the laboratory TAT. ${ }^{11}$

Along with customer satisfaction, decreased laboratory TAT also improves the efficiency of ED work by allowing the early diagnosis, treatment, patient discharge and provides a way of cost cutting. A simulation study conducted by Storrow et al suggested improved ED efficiency and reduced length of stay following the reduction in TAT. ${ }^{12}$ According to Ling Li et al, ED length of stay was directly influenced by laboratory TAT. ${ }^{13}$ Gill et al also showed a positive correlation between laboratory TAT and length of stay. ${ }^{14}$

Regular monitoring of TAT itself acts as a very useful tool to improve the routine TAT as it aids to uncover and correct the unrecognised inefficiencies in laboratory operation. According to Peller GA et al daily monitoring and plotting of TAT in the form of freckle plot serves as a useful quality improvement tool. ${ }^{15}$

\section{Conclusion}

In our laboratory, we have achieved a significant reduction in the TAT of ED samples by introducing few simple modifications in the routine workflow which can be of a great benefit to patients. Our study has highlighted the power of analytical phase intervention in the form of reallocation of resources and staff awareness in effectively reducing the TAT. The study also proved the usefulness of having separate colour coded ERF in early identification, segregation and processing of ED samples.
Limitation: Long-term sustainability of the effects of intervention was not assessed periodically due to the lack of resources.

\section{Clinical Significance}

Reduced laboratory turnaround time of ED investigations is clinically significant because along with customer satisfaction it also improves the efficiency of work by allowing the early diagnosis, treatment, patient discharge and provides a way of cost cutting.

\section{References}

1. Hawkins RC. Laboratory Turnaround Time. Clin Biochem Rev 2007; 28:179-194.

2. Moyo K, Porter C, Chilima B, Mwenda R, Kabu M et al. Use of laboratory test results in patient management by clinicians in Malawi. Afr J Lab Med2015;4(1): 1-11.

3. Al-Talib M and Leslie I. Speeding up laboratory test reporting in Medical Emergency and Cardiac Arrest calls: a quality improvement project. BMJ Quality Improvement reports 2017; 6:1-7.

4. Bruel AV, Thompson MJ, HajHassan T, Stevens R, Moll H et al. Diagnostic value of laboratory tests in identifying serious infections in febrile children: systematic review. BMJ 2011; 342:1-11.

5. Goswami B, Singh B, Chawla R, Gupta VK and Mallika V. Turn Around Time (TAT) as a Benchmark of Laboratory Performance. Ind J Clin Biochem 2010; 25(4): 376-379.

6. Ong, D.C. A Primer to Bootstrapping; and an overview of do Bootstrap. Department of Psychology, Stanford University, Palo Alto; 2014

7. Gjolaj LN, Gari GA, Olier-Pino Al, Garcia JD and Fernandez GL. Decreasing Laboratory Turnaround Time and Patient Wait Time by Implementing Process Improvement Methodologies in an Outpatient Oncology Infusion Unit. Journal of oncology practice 2014; 10(6):380382.

8. Umut B and Sarvari PA. Applying lean tools in the clinical laboratory to reduce turnaround time for blood test results. International Journal of Advances in Science Engineering and Technology 2016; 4(3):164-169.

9. Angeletti $S$, Cesaris MD, Vitali $S$, Bernardini $S$ and Dicuonzo G. Monitoring and improvement of intralaboratory turnaround time at the university hospital Campus Bio-Medico in Rome. Biochimica clinica, 2014;38:115-120.

10. Chien T, Lu J, Kao J, Cheng Y and Lee1 Y. Evaluation and Improvement Strategy of Analytical Turnaround Time in the Stat Laboratory. J Formos Med Assoc 2007; 106(7): 558-564.

11. White BA, Baron J, Chang $Y$ and Camargo CA. Applying lean methodologies reduces emergency department laboratory turnaround times. Annals of emergency medicine 2014; 64(4S): S9.

12. Storrow AB, Zhou C, Gaddis G, Han JH, Miller K et al. Decreasing Lab Turnaround Time Improves Emergency Department Throughput and Decreases Emergency Medical Services Diversion: A Simulation Model. Acad Emerg Med 2008; 15(11):1130-1135.

13. Li L, Georgiou A, Vecellio E, Eigenstetter A, Tooul G et al. The Effect of Laboratory Testing on Emergency Department Length of Stay: A Multihospital Longitudinal Study Applying a Cross-classified Random-effect Modeling Approach. Acad Emerg Med 2015; 22(1): 38-46.

14. Gill D, Galvin S, Ponsford M, Bruce D, Reicher J et al. Laboratory sample turnaround times: do they cause delays in the ED? J Eval Clin Pract. 2012;18(1):121-127.

15. Pellar TG, Ward PJ, Tuckerman JF and Henderson AR. The Freckle Plot (Daily Turnaround Time Chart): A Technique for Timely and Effective Quality Improvement of Test Turnaround Times. Clin Chem 1993; 39(6): 1054-1059. 